\title{
HUMAN MICROCHIP IMPLANTATION
}

\author{
Charles Smith (I)
}

\begin{abstract}
Radio Frequency Identification (RFID) has generated a lot of attention in recent years as a method of replacing the barcode. This paper will begin by examining some of the origins of RFID technology. From there it will look at several applications of RFID technology within the health care industry. Next, privacy and legal issues will be examined. Lastly the result of a survey of local students
\end{abstract}

Keywords: Microchip Implantation, Privacy, RFID, Electronic Product Code (EPC)

(I) Mesa State College. cesmith@mesastate.edu. Phone Number (970)254-0443 


\section{Introduction:}

RFID technology had humble beginnings in WWII to identify Allied airplanes. From there, RFID technology was used to track nuclear material and animals. The technology took another jump and is currently used to track items through the supply chain.

A RFID chip is a microchip that can transmit a static identifier or serial number for a short distance. Research is being done to help people medically with RFID technology. Microchips can help advance prosthetics use, help people hear better, or possibly even help paralyzed people move. The technology is available and being used to implant people with microchips. Some issues arise with implanting people with microchips though. Issues include, being able to track a person's previous and current location, their purchasing habits, legal and privacy concerns, as well as hacking their personal and financial information.

\section{Problem:}

There are both potential problems and benefits associated with human microchipping. One problem is that a person's privacy could be severely infringed upon. This could happen because the person's movements, both physically and financially, could be tracked. Personal data about a person could be sold or hacked into. A third potential problem could be who would have access to the information, and who stores the information.

There are potential health problems as well. For example Nonlonizing Radiation from microwave radio frequency and magnetic fields could cause various health issues. (Covacio, 2003) A potential benefit could include storing a person's complete medical history, or at the bare minimal the drugs that they are taking or are allergic to. (Fuhrer \& Guinard, 2006)

\section{History:}

RFID technology began in World War II with an "Early Identification Friend or Foe (IFF) systems where it was possible for Allied fighters and anti-aircraft systems to distinguish their own returning bombers from aircraft sent by the enemy." (Garfinkel \& Holtzman, 2005, p. 15) Then shortly after WWII, Henry Stockman, who was an engineer, had a paper published called "Communication by Means of Reflected Power" where he came up with the idea for passive RFID chips. (Garfinkel \& Holtzman, 2005, p. 16) In 1960, RFID technology was used to identify and monitor hazardous material and nuclear power. (Miller, 2007)
The first commercial application with RFID was the Electronic Article Surveillance for anti-theft purposes. There were early attempts in 1960 in RFID technology. For example, in 1963 Robert Richardson created remotely activated radio frequency powered devices. In $1968 \mathrm{~J}$. H.Vogelman was working on Passive Data Transmission Techniques Utilizing Radar Beams. Then in 1969 Otto Rittenback was working on Communication by Radar Beams.

Even more work on RFID chips started in the 1970's. In 1972, Kriofsky and Kaplan filed a patent application for an "inductively coupled transmitter-responder arrangement."(Garfinkel \& Holtzman, 2005, p. 16) The system had two separate coils, one to receive power and another one to send the signal. In 1979 Beigel filed a new patent combining the two coils, which allowed for the small size of an RFID chip. The tag could be implanted into any item that is for sale. Chips would hold a single bit, if the bit was turned off the alarm system wouldn't go off, but if the bit was left on then an alarm would go off. Jechlitschek, 2006) In the 1970's and 1980's researchers, universities, and even government agencies worked on RFID technology to make the chips smaller, cheaper, and able to transmit the frequency farther. Each tag could be preprogrammed with some basic functions that may include:

- "A kill command- disables the tag permanently

- A sleep command- the tag no longer responds to queries, except upon receipt of a wake command.

- Read- sends ID to a reader

- Write- takes information from reader and stores it." (Francom, 2007, pg. 337)

In the 1970's the Lawrence Livermore Laboratory created a handheld receiver that could receive encoded messages from the chip. It is said that the technology was secure because it was so obscure.

One of the first uses for RFID technology was in the agriculture industry. Tags were implanted into cattle. There were two main reasons for implanting cattle. The first was approximately onethird of the hide was damaged when the cattle were branded, which meant less usable leather. The chips allowed the farm to track and distinguish their animals from other people's cattle with the instead of branding. Second was for automated feeding. With the chip, automated feeding was allowed without worrying about if the cattle would be overfed. (Miller, 2007)

RFID went from implanting animals to the railroad industry. Originally railroad cars were tracked with barcode on the side 
of the cars but were difficult to read in adverse weather or if the barcodes became dirty. RFID chips were put on cars and antennas were put along tracks. With the RFID chips railroad companies could better track the cars, which in turned help with scheduling of the cars and whatever they needed to ship, as well as reducing congestion along the tracks. (Miller, 2007)

In the 1980's RFID took off with automated payments. For example, Norway and several U.S. states used RFID chips for toll collections on the road, with systems like EZ-Pass. The toll collection system in turn led to collection systems for the ski industry, purchasing gas, money cards, and more. (Jechlitschek, 2006)

\section{Health Care:}

Within the medical field there are different ways that RFID can be incorporated. For example RFID chips can be used to track equipment within a hospital. Plus, with RFID tags drug manufacturers can better manage the drugs through the supply chain, for the simple fact "that the pharmaceutical industry loses between $\$ 10$ and $\$ 30$ billion per year to counterfeit versions of drugs.'(Castro \& Wamba, 2007, p. 4)

Pharmaceutical companies can embed RFID chips into prescription bottle caps, cases or pallets to track the drug through the supply chain. For example, CVS Corporation, in 2002, teamed up with the Auto-ID center at MIT and started a project in 2003 called "Jump Start," (Hildebrandt \& Meints, 2006) for a full scale trial of RFID implementation. The following list contains some of the main reasons that CVS wanted to include RFID:

- "Pharmaceuticals are high valued goods,

- Pharmaceuticals may have a long shelf life (depending on turnover rate),

- In the United States tamper-proofness of pharmaceuticals in the logistic chain and the shops is an issue since the Tylenol scandal in 1982, where Tylenol was adulterated with cyanide and as a consequence a number of consumers died. CVS is using RFID on a per item basis. CVS is tagging both individual bottles and the packing that the bottles are shipped in. There are a number of potential improvements in processes at CVS. Some include:

- Improvement of drug management at the manufacturer and in the distribution centres of CVS; errors in the delivery such as wrong types or numbers of drugs can be detected easily;

- Improvement in drug management in the stores; the central systems know how many goods are left in the smart shelves even in cases where they are at the wrong place in the shelf (supply management);

- Improved handling of outdates, recalls, returns and damages;

- EPC stored on RFID can be used to detect certain types of mistakes or manipulations of drugs for example in cases where already used or cloned RFID tags are used." (Hildebrandt \& Meints 2006, pg. 24-25)

Wristbands with an RFID chip embedded can help hospitals with patient safety requirements. Medical personnel can read the chip to get instant access to the patients' medical history, such has medication allergies, medications prescribed and dosage, and specimen results. (Castro \& Wamba, 2007)

Hospitals can also use RFID to implant chips into people with forms of dementia or newborn babies, that way if the people leave a certain area, it will trigger an alarm alerting the staff. Medical staff would be able to react and stop the person before they left the hospital or specific ward. The person with dementia would not be able to leave the ward, or a baby would not be abducted. With an RFID chip there would be a much smaller chance of babies being swapped by mistake.

An added benefit is an embedded chip or RFID wristband can include both patient information, allergies, and medication the patient is currently taking or medication a doctor will prescribe to the patient. With the wristband, an RFID reader, and a wireless connection, a doctor or nurse can pull up the patient's information, or even update the patient information in real time. Some benefits of this include less patient mix-up, being able to access the patient information in real time, and fewer mistakes due to human error in entering the data at a nurse's station. A scanner device could be used to enter in the updated information. Productivity would increase since the doctors and nurses would not have to go to a computer to enter in the updated information. This would allow doctors and nurses can see more patients.

There is a wide array of medical uses for microchip implants. One example is using the implants with prosthetics. An RFID chip could be placed on top of the brain to improve movement or functionality of the prosthetic. With the implant, the person would have both input and output capabilities. The downside to the brain implant is that the operation is more invasive. As an alternative, doctors could place an electrode on the limb itself that would act on electrical impulses from the brain. The operation is less invasive, but the electrode would have very limited input capabilities. 


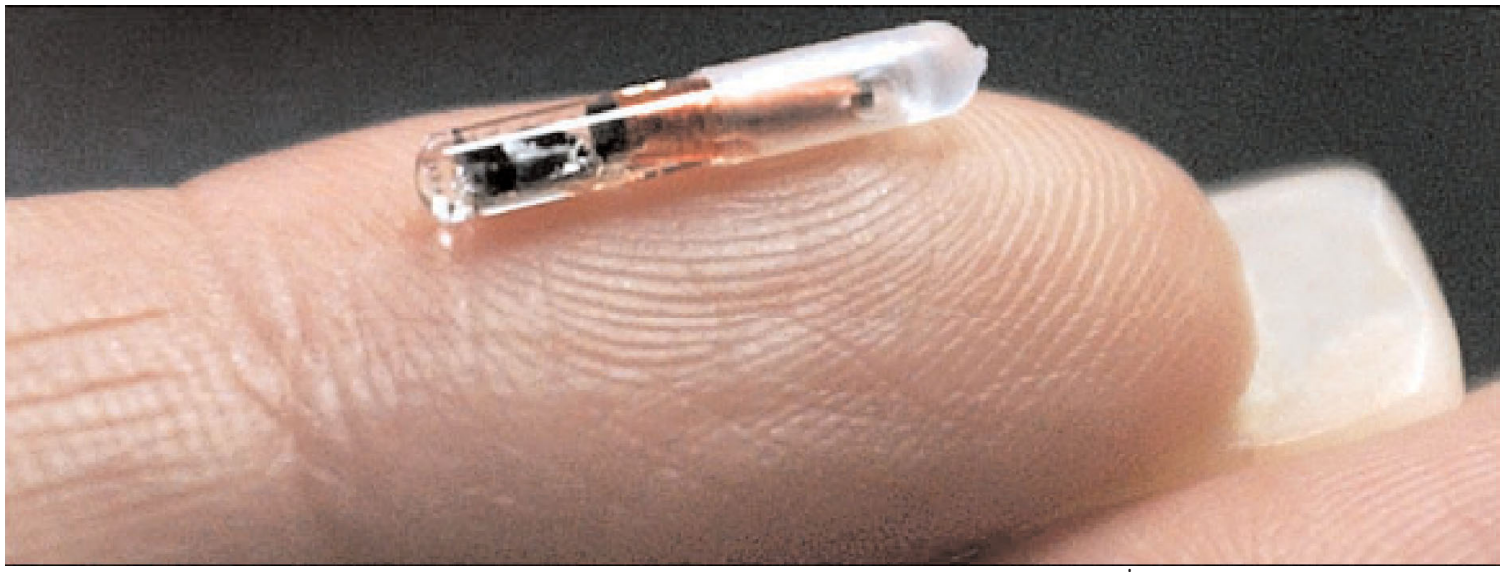

Figure I. Figure of an RFID Chip(Feder \& Zeller, 2004)

Myoelectrical limbs are another possible use of RFID. With a myoelectrical limb, the limb can detect and amplify electrical signals. The limb then deciphers the signals and movements of the muscles to determine how the limb should react. For example the signals indicate if the limb should move horizontally or vertically, or if the hand should open or close.

A third possibility is to control prosthetic limbs or give commands for computers is with electroencephalogram (EEG). Electrodes are placed on many different areas of the brain that are used to sense activity from the brain. When the user is trained they could move the cursor across the screen. With this application, patients have to be trained to control their neural activity to achieve the intended result. With neuroelectronic interface chips that are implanted directly onto the top of the brain, the users don't have to be trained. The chip can translate the neural activity into the desired result.

One example of the Neuroelectronic Interface being used is with a paraplegic named John Nagle. "Nagle showed an ability to perform a number of tasks with his mind: control a TV, move a mouse cursor on a screen, and command an artificial hand to open and close grip." (Chan, 2007, p. I3)

There are limitations at this time for the Neuroelectronic Interface. The first is the amount of neurons available for the device. The less amount of neurons there are, the less likely that the device will work. Also the devices are not wireless, which means that there are wires coming out of the brain.

There are health risks associated with being implanted with a microchip. Some health issues include "adverse tissue reaction, migration of implanted transponder, electromagnetic interference, electrical hazards, and magnetic resonance imaging incompatibility. " (Gad, 2006, p. I)

\section{Laws:}

There are legal and legislative issues as well dealing with RFID technology. Even though the scanner that reads the sensor has to be close to the body to read the chip, there still is the possibility of identity theft. Having the chip implanted has been compared to carrying your Social Security Number under your skin.

Legal issues with neuroelectronic interface enhancements have risen as well. Currently there is no governmental agency that control neuroelectronic interface enhancements. As it stands today, the one agency that could control RFID and neuroelectronic interface devices is the FDA, because it has broad regulatory jurisdiction over medical devices. The FDA defines a medical device as "the diagnosis ... treatment or prevention of disease" or as something used "to affect the structure or any function of the body."(Chan, 2007, p. 24) Within the FDA, the Center for Devices and Radiological Health (CDRH) regulates the approval and manufacturing of all medical devices within the U.S. which includes the post market period.

Issues lawmaker's would have to keep in mind could include:

- What information should be placed on a device to be able to identify the person

\footnotetext{
* Figure Description: Figure one is a picture showing the size of an RFID chip.
} 
- Who would have access to the personal information

- Who would be able to read the information with a scanner

- What would be a punishment with people stealing the information with a scanner

- At what level of government should the laws should be enacted

- Local laws may be too narrow to include all the problems with RFID

- National laws may be too broad, or be too cumbersome for business' and stifle innovation

- Constitutional restrictions apply only to governmental actions but not to private enterprises or individuals.

One thing that lawmakers need to keep in mind is to enact laws that encompass both the government and private sectors. Take for example the Privacy Act of 1974," the most comprehensive U.S. law pertaining to privacy applies only to government actors. Therefore, private corporations are not bound by the fair information practices, open-access rules, and data-ownership principles embodied in the Act." (Peek, 2006 pg. I 36)

The U.S. Federal Government does not have a comprehensive privacy law; instead there are privacy laws for specific industries. For example there is the Fair Credit Reporting Act (FCRA), the Graham-Leach-Bliley Act (GLBA) for financial data, and the Health Insurance Portability and Accountability Act (HIPAA). The Fourth, Fifth, and Fourteenth Amendment dealing with privacy are not much help either. At present the Supreme Court has not interpreted the Fourth Amendment to include things like "GPS, RFID, or real time cell site monitoring." (Herbert, 2006, pg. 4I7) There are "four reasons given by the ACLU and Consumers Against Supermarket Privacy Invasion and Numbering (CASPIAN) on the ethical concerns of RFID:

I. The tags are hidden and unknown.

2. Tags provide an id system of every item purchased, thus allowing a universal product registration system.

\section{Aggregation of massive personal amounts of data.}

4. Allows tracking of individuals.” (Francom, 2007, pg 337)

Part of the problem is security, confidentiality and data integrity. The system storing the data and the tag have to be secure. The person's information cannot be disclosed to unauthorized people or entities. There has to be data integrity or that the data has to be complete and consistent. Federal, state, or local laws have to ensure the security, confidentiality, and integrity of the personal information.

\section{Conclusion:}

This paper presented the timeline for RFID advancements. It also discussed how RFID can be used within the health care industry. Privacy issues and laws surrounding RFID were looked at. There is a need for more laws dealing with RFID to protect privacy and protecting consumer's information. The hard part will be to enact laws that protect consumers while not stifling innovation of RFID technology. From the survey results a majority of the respondents believe that if people got implanted with a RFID chip, that their right's to privacy, and not to incriminate themselves would be violated. A majority of the respondents would not get implanted with an RFID chip either.

\section{References:}

CASTRO, L., Wamba, S.F. (2007) An Inside Look At RFID Technology. Journal of Technology and Innovation, Volume 2, Issue I. Pg. 4.

CHAN, E. (2007) The FDA and the Future of the Brain-Computer Interface: Adapting FDA Device Law to the Challenges of Human-Machine Enhancement. John Marshall Journal of Computer \& Information Law Volume 25 Issue I. Pg. I3, I6, 24. http://works.bepress.com/eric_chan/I/. Retrieved II-02-07

COVACIO, S. (2003) Technological Problems Associated with Subcutaneous Microchips for Human Identification (SMHId). Informing Science.

FEDER, B., Zeller Jr., T. (2004) Identity Badge Worn Under Skin Approved for Use in Health Care. New YorkTimes. Retrieved I I-30-07.

FRANCOM, Joseph (2007) RFID:A Survey Of Ethical And Privacy Concerns. Issues in Information Systems.V3 Issue 2i. Pg. 337.

FUHRER, P., Guinard D. (2006) Building a Smart Hospital using RFID technologies. European Conference on eHealth. Pg. 5. http://diuf.unifr.ch/people/guinard/docs/wp_smarthospital.pdf. Retrieved 10-28-07.

GAD, L. (2006) Human Microchip Implantation. Wisconsin Legislative Reference Bureau. Pg. I. www.legis.state.wi.us/lrb. Retrieved 9-28-07. 
GARFINKEL, S., Holtzman, H. (2005) Understanding RFID Technology. Pg. I5-16. Garfinkel Book

HERBERT,W. (2006) No Direction Home:Will The Law Keep Pace With Human Tracking Technology to Protect Individual Privacy and Stop Geoslavery? I/S:A Journal Of Law And Policy Volume 2 Issue 2. Pg. 4I7. Retrieved I0-I5-07.

HILDEBRANDT, M., Meints, M. (2006) FIDIS Future of Identity in the Information Society. pg. 24-25. FIDIS Consortium.

JECHLITSCHEK, C. (2006) A Survey Paper on Radio Frequency Identification (RFID) Trends. Pg. 2. http://www.cse.wustl.edu/ jain/cse574-06/ftp/rfid/index.html. Retrieved 10-15-07

MILLER, S. (2007) What is RFID? (Dissertation, Purdue University, 2007) Retrieved 08-26-2007

PEEK, M. (2006) Information Privacy and Corporate Power:Towards a Re-Imagination of Information Privacy Law. Seton Hall Law Review Vol. 37 Issue 127. Pg. I36. Retrieved 0I-09-2008. 


\section{Appendix A}

\section{RFID Implantation Questionnaire}

You are being asked to complete this survey as part of a of a research study by a Computer Information System student. Participation is this survey is voluntary. The survey is anonymous. Please do not put your name anywhere on this survey. While we would like you to answer all of the questions on this survey, you are not required to do so. You may end your participation in this survey at any time. By completing this survey, you give your consent to participate in this research project. Your responses will be held confidential. Data will be reported only in aggregate.

A Radio Frequency Identification (RFID) chip can be implanted into people. If an RFID chip is implanted, personal information can be collected and stored, some examples include previous or current location, and medical history.

\section{What is your level of knowledge about RFID Technology?}
Not Familiar I
2
3
4
5 Very Familiar

Please rank who should control the data collected? (Rank I-4)

_ Governmental agency

Company who collected data

Implanted person

Data should not be collected at all

\section{Please rank who should have access to the data? (Rank I-4)}

Governmental Agency

Implanted person

\section{Company who collected data}

Data should not be collected at all

Please rank how law enforcement should be able to access the data? (Rank I-4)

Court order

Company turns over data

Implanted person consent

_Data should not be accessed at all

\section{Would an RFID chip violate a person's right to privacy? (Check One)}

$$
\text { Yes_No }
$$

Would an RFID chip violate a person's right not to incriminate themselves? (Check One)

Y Yes_No

\section{Should RFID chips be implanted in people? (Check One)}

Yes No

\section{Would you get implanted with an RFID Chip? (Check One)}

Yes

No

What is your major?

Age

\section{Gender (Circle One)}

Male Female

Thank you for your time and cooperation. Please return to HI 06 or person administering survey. 


\section{Appendix B}

\section{Survey Statistics}

The survey respondent's are a sample population of business faculty and students. From the survey though it cannot be determined the factors contributing to the way the questions were answered. Possible factors could familiarity with technology in general, education level, or degree earned, to name a few.

From the respondents, the average age of professors is 50 years old, and for students the average age is 27 years old.

\section{Question I.What is your level of knowledge about RFID Technology?}

On average professors ranked 3 or being in the middle on their familiarity on RFID. Students, males and females ranked 2 on being familiar with RFID.

\section{Question 5. Would an RFID chip violate a person's right to privacy?}

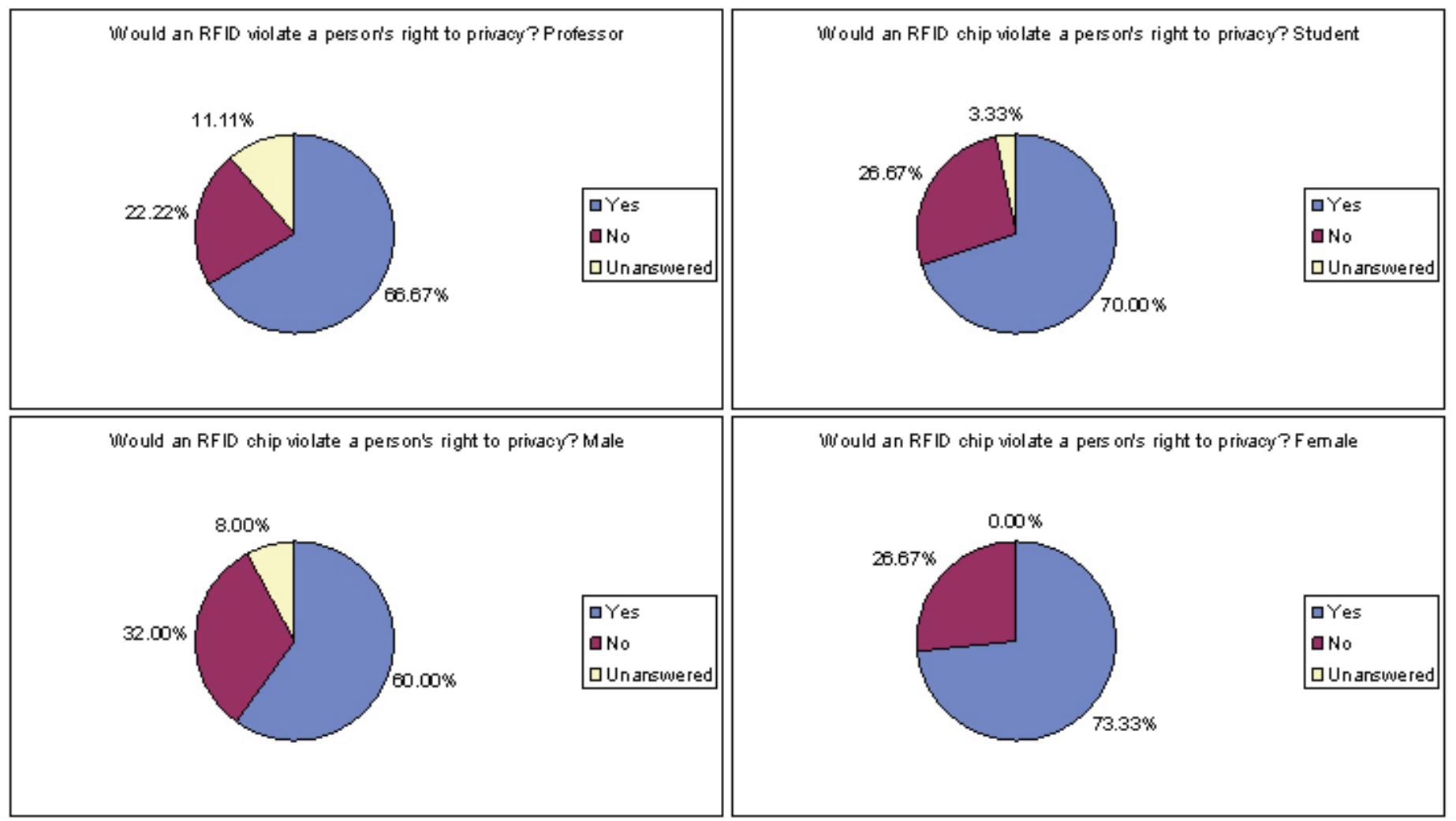

A majority of the survey population believes that implanting people with RFID chips would be an invasion of their privacy. Again from the survey though it cannot be determined the factors contributing to the knowledge of RFID. Possible factors could familiarity with technology in general, education level, or degree earned, to name a few.

\section{Question 6. Would an RFID chip violate a person's right not to incriminate themselves?}




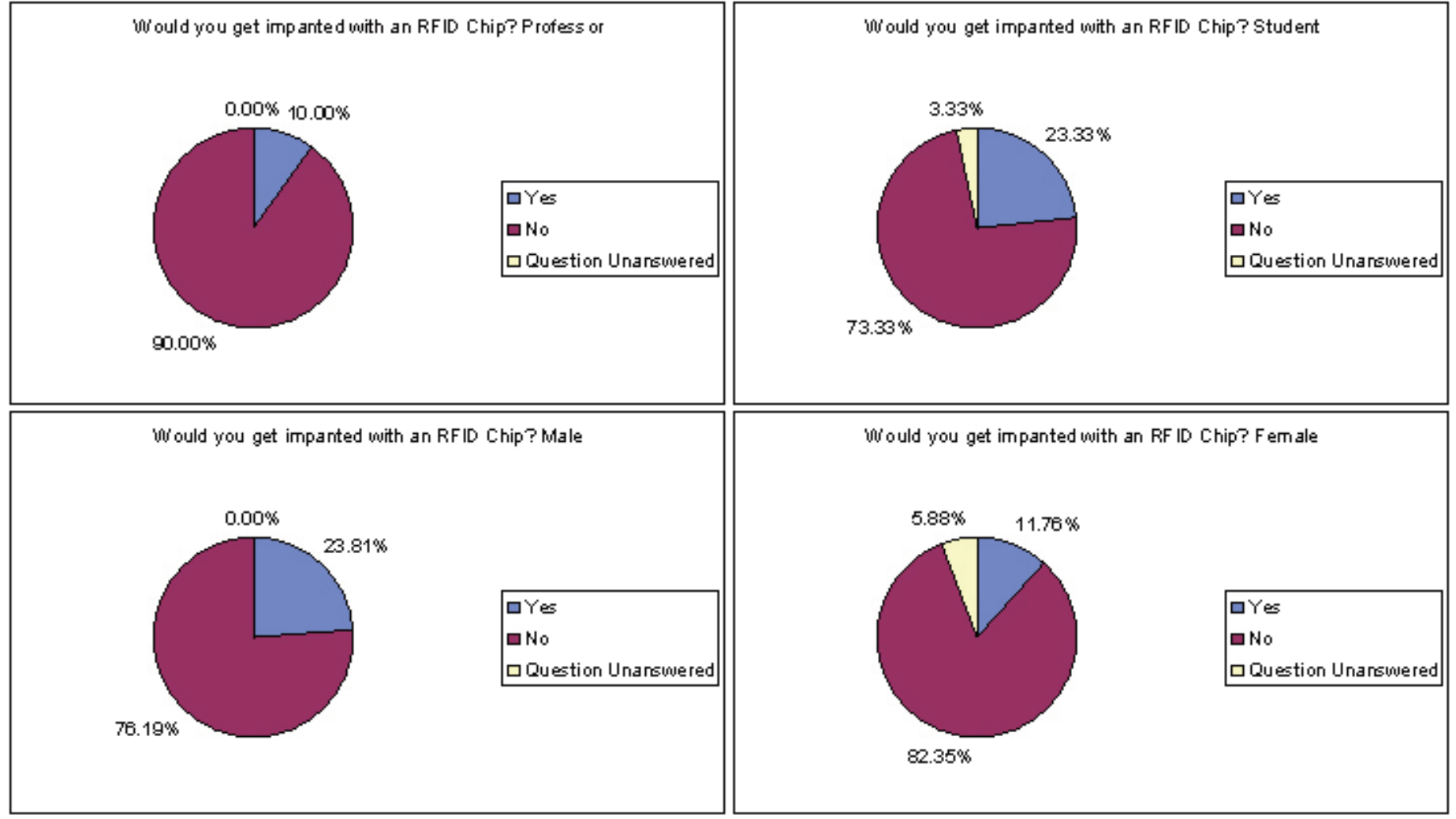

Over half of the professors and students believe that RFID technology would incriminate themselves. Where as there is a bigger split between males and females. Where more females believe that the technology would incriminate them.

\section{Question 7. Should RFID chips be implanted in people?}

Should $\mathrm{RFID}$ chips be implanted in people? Profess or


A majority of the survey population believes that people should not be implanted with RFID chips. The student population was the only ones that are nearly evenly split between answering yes and no on the question.

\section{Question 8. Would you get implanted with an RFID Chip?}

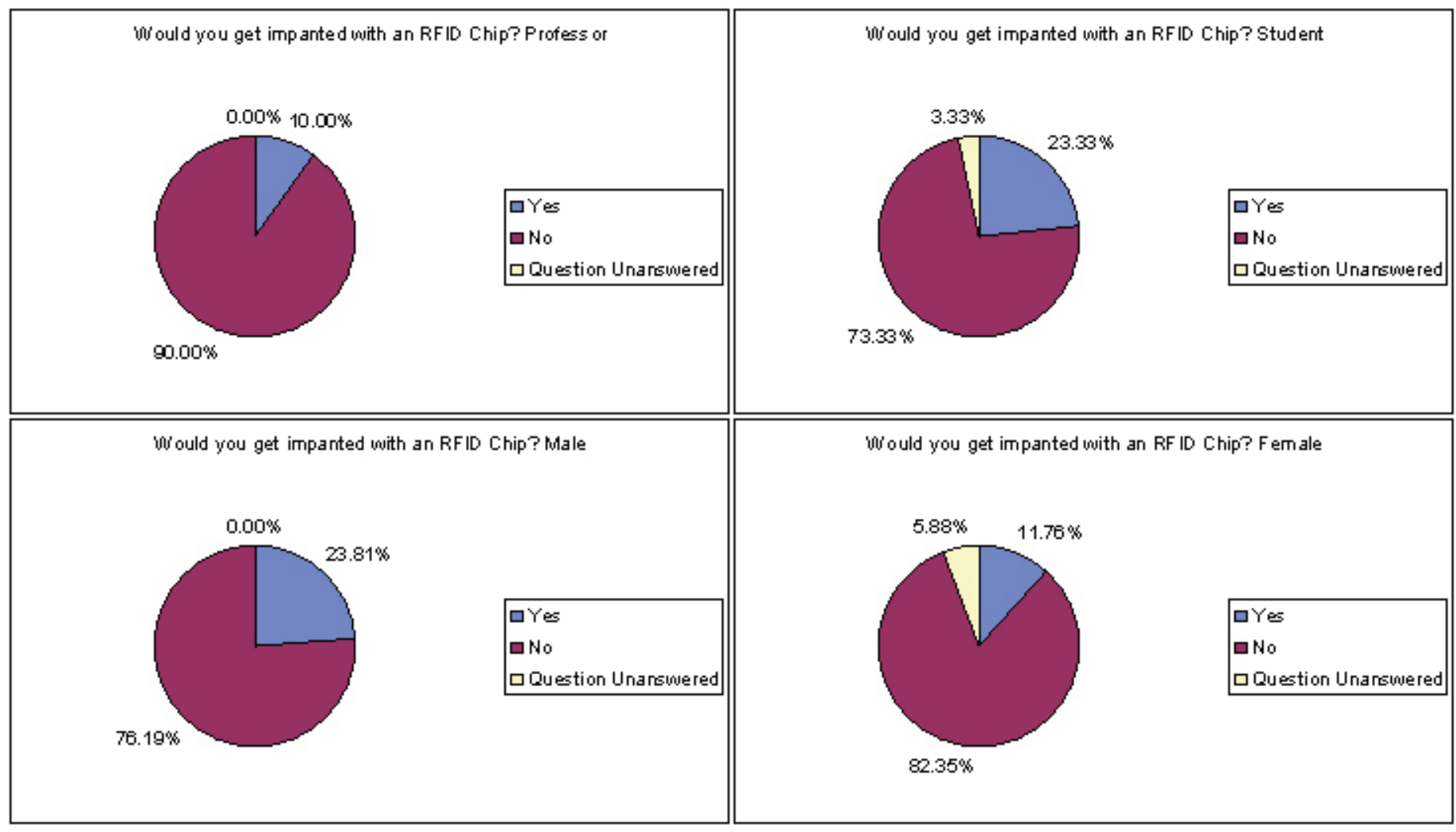

A majority of the survey respondents would no get implanted with an RFID chip. Even with professors being more familiar with the technology they are more likely not to get implanted with a chip. 